\title{
Desperately seeking embryos (from leftover oocytes)
}

David F. Albertini

Published online: 17 July 2010

(C) Springer Science+Business Media, LLC 2010

Keywords Controlled ovarian stimulation · Immature oocytes

While searching for a means of optimizing the number of embryos available for transfer, ARTs, as we know, have come to rely upon controlled ovarian stimulation (COS) to produce as many mature oocytes as possible. Cognizant of the power of gonadotropin preparations that evolved from natural sources, to the next generation of recombinant proteins, more, bigger follicles were excavated in search of the precious pearl: an oocyte capable of producing a term gestation following IVF or ICSI. Suspicions have been raised repeatedly as to the impact of COS on the overall yield of high quality oocytes that would be needed to generate high quality embryos - that is those capable of making a baby. Are such oocytes, physiologically destined to the dead end known as atresia, capable of being rejuvenated and repurposed into a fate consonant with the rules of pregnancy? And what effect, if any, would the pharmacological acceleration of follicular development have on the apparently methodical pace of procrastination that the oocyte takes during its journey through the final stages of oogenesis? Unfortunately, as reported in the paper by Reichman et al in this issue, up to $30 \%$ of these fail to complete the run to "meiotic "maturity required to get onto the embryo bandwagon in concert with the daily operations of an ART facility.

Capsule The clinical utility of in vitro matured oocytes retrieved after controlled ovarian stimulation is unlikely.

D. F. Albertini $(\bowtie)$

University of Kansas Medical Center,

Kansas City, KS 66103, USA

e-mail: dalbertini@kumc.edu
This population of oocytes, laggards as it were, deserves such a classification because at the time of retrieval such oocytes exist in either germinal vesicle or meiosis I stages. Historically, these immature oocytes have assumed a special importance for patients unfortunate enough to have yielded few mature oocytes after COS. Indeed, some patients may yield only immature oocytes! For them, a second opportunity to achieve maturity would increase the chances of having embryo transfer and a pregnancy. This has been one of the hopeful dimensions of human ARTs, based on the prospect of allowing these laggards to catch up with their mature sisterhood after prolonged culture. "Better late than never at all" designates this form of human oocyte IVM.

What to do, then, with these immature oocytes obtained after $\mathrm{COH}$ ? This is an especially valid question when posed in the context of human oocyte research. Could this material serve to fill the void we now have for optimizing techniques such as oocyte cryopreservation and IVM? Until now there has been little doubt that such oocytes do "mature" after prolonged culture, but the likelihood that these oocytes would exhibit some of the initial qualities that are expected to follow IVF or ICSI has not been fully determined. What the study by Reichman and associates now alerts us to is in some ways predictive of the "dark side" of COS, but in other ways raises awareness as to the lack of fundamental knowledge we now have concerning the biology of the human oocyte. To quote Christopher Marlowe, "there is no sin but ignorance."

Reichman and colleagues undertook an analysis of 234 in vivo matured human oocytes and compared their postICSI developmental performance to 263 matched oocytes that were evaluated following a 24-hour period of in vitro maturation. As in previous studies, oocytes used for IVM 
were distinguished from their in vivo-matured counterparts in several critical ways. First, these oocytes clearly were retrieved from follicles that did not support their maturation after COS (six different protocols were tracked) as they were arrested in either the GV or M1 stages of meiosis. Second, staging for meiotic status and access for subsequent ICSI requires removal of any adherent cumulus cells, meaning that whatever degree of maturation these oocytes could achieve in vitro was done in the absence of supportive somatic cells. Finally, the medium employed for IVM was supplemented with recombinant FSH and LH $(75 \mathrm{mIU} / \mathrm{mL}$ each), as recommended by the commercial providers, and created a remarkably different microenvironment from that experienced by oocytes that matured within the follicle prior to their retrieval. Given this, the question has been can oocytes matured under these conditions produce developmentally competent zygotes after ICSI? The answer: No.

Tracking these oocytes with respect to their degree of maturation in vitro revealed that, in fact, only $48 \%$ were able to reach $\mathrm{M} 2$, and the vast majority of these were derived from oocytes that were already at M1 when subjected to culture. Notably, of the GV-stage oocytes placed in culture, only $35 \%$ matured to M2 (compare to $78 \%$ if IVM was initiated at M1 stage). The first and hardly surprising message is that, given the heterogeneous follicular source of these oocytes, nearly half were not only unable to initiate maturation in vivo, but they were also unable to do so in vitro. By comparison, if maturation was initiated in vivo, there was a greater likelihood of completing this process when subsequently cultured.
More telling and critical to the clinical utility of these oocytes was the assessment of their developmental progression analyzed in day 2 embryos produced by ICSI. For all three parameters reflecting embryo quality that were evaluated (cleavage rate, fragmentation, blastomere symmetry), clear evidence for developmental delays and aberrations was noted for the IVM oocytes, relative to their sibling IVO oocytes. The fact that no pregnancies were obtained after transfer reinforces this baseline data in documenting fundamental disturbances in cell-cycle timing and progression that were likely to have been compounded by severe aneuploidies and mosaics, had these embryos been subjected to PGS.

There are two important lessons this study underlines: One, with the drive to optimize IVM for human oocytes as an efficient and safe ART, the use of such "spare" oocytes for research applications in this area is both superfluous and likely to be very misleading. This fact emphasizes that future research must rely upon intact COCs subjected to pharmacological inhibitors, so as to harness the synchrony of cytoplasmic and nuclear maturation in the context of somatic cell support with appropriate human materials and/or animal models. Two, the clinical utility of this material is abysmal and, given that the characteristics shown by these embryos, is sentinel for major genetic alterations. One wonders, then, whether a conservative and clear statement should be made to limit transfer of such embryos to truly exceptional cases.

Stay tuned as we continue to cover the evolution of ARTs in the context of contemporary science. 